\title{
Pilot-Symbol Assisted Power Delay Profile Estimation for MIMO-OFDM Systems
}

\author{
D.Srinivasa Rao *, S.Venkata Swamy, B.Praveen Chakravarthy \\ Student M.Tech /Dept.Of. Ece /Sistam College/jntuk/Srikakulam,A.P, India \\ Professor\& Head/Dept .Of. Ece/Sistam College/jntuk/Srikakulam,A.P, India \\ Assistant Professor/ Dept.Of. Ece /Sistam College/jntuk/Srikakulam,A.P, India
}

\begin{abstract}
This letter proposes a power delay profile (PDP) estimation technique for linear minimum mean square error (LMMSE) channel estimator of multiple-input multiple-output orthogonal frequency division multiplexing (MIMO-OFDM) sys-tems. For practical applications, only the pilot symbols of all transmit antenna ports are used in estimating the PDP. The distortions caused by null subcarriers and an insufficient number of samples for PDP estimation are also considered. The proposed technique effectively reduces the distortions for accurate PDP estimation. Simulation results show that the performance of LMMSE channel estimation using the proposed PDP estimate approaches that of Wiener filtering due to the mitigation of distortion effects.

Index Terms: Channel estimation, power delay profile, MIMO, OFDM, 3GPP-LTE
\end{abstract}

\section{Introduction}

MULTIPLE-INPUT multiple-output orthogonal frequency division multiplexing (MIMO-OFDM) is one of the most promising techniques for wireless communication systems, including the 3rd Generation Partnership Project Long Term Evolution (3GPP LTE) [1], [2] and IEEE 802.16 (WiMAX). MIMO-OFDM provides a considerable performance gain over broadband single-antenna systems by obtaining the spatial diversity or multiplexing gain [3], [4]. Most receiver techniques of MIMO-OFDM systems are designed with the assumption that channel state information (CSI) is available, in order to achieve the maximum diversity or multiplexing gain [5]-[7]. The performance gain depends heavily on accurate channel estimation, which is crucial for the MIMO-OFDM systems.

The pilot-aided channel estimation, based on the linear minimum mean square error (LMMSE) technique, is optimum in the sense of minimizing mean square error (MSE) when the receiver knows the channel statistics [8]. To obtain the frequency domain channel statistics at the receiver, power delay profile (PDP) estimation schemes have been proposed [9], [10]. These schemes are based on the maximum likelihood (ML) estimation by taking advantage of the cyclic prefix (CP) segment of OFDM symbols. However, the ML PDP estimators require very high computational complexity for obtaining an accurate PDP.

Another approach for improving the performance of LMMSE channel estimation employs an approximated PDP (i.e., uniform or exponential model) with the estimation of second-order channel statistics, which are mean delay and root-mean-square (RMS) delay spread [11]. The channel delay parameters are estimated using pilots with low computational omplexity. Therefore, the LMMSE channel estimator with the approximated PDP is appropriate for practical applica-tions such as a WiMAX system. However, the performance degradation is caused by both the correlation mismatch and the estimation error of delay parameters.

To reduce the mismatch in the frequency domain, we propose a PDP estimation technique for the LMMSE channel estimator of MIMO-OFDM systems. For practical applica-tions, the proposed technique uses only the pilot symbols of all transmit antenna ports to estimate the PDP with low computational complexity. In addition, the proposed technique effectively mitigates the distortion effects, incurred by null subcarriers and an insufficient number of estimated channel impulse response (CIR) samples. Simulation results show that the performance of LMMSE channel estimation with the proposed PDP estimate approaches that of Wiener filtering. 


\section{System Model}

The system under consideration is a MIMO-OFDM sys-tem with $\mathrm{C}$ transmit and $\mathrm{D}$ receive antennas, and $<$ total subcarriers. Suppose that the MIMO-OFDM system transmits $<_{\mathrm{c}}$ subcarriers at the central spectrum assigned for data and pilots with $<-<_{\mathrm{c}}$ virtual subcarriers, in order to control interferences with other systems. The CIRs corresponding to different transmit and receive antennas in MIMO systems usually have the same PDP [12].

Let $\_n\left[R_{n}, T_{n}\right]$ be the pilot subcarrier for the ath trans-mit antenna at the $T_{n}$ th OFDM symbol, which is a QPSK modulated signal from known sequences between the trans-mitter and receiver. We assume that the pilot subcarriers are distributed over a time and frequency grid as in Fig. 1 , to preserve the orthogonality of pilots among different transmit antennas. $R_{n} \in \mathcal{F}_{n}$ and $T_{n} \in \_n$ represent the index sets for the pilot subcarriers of the ath antenna port in the frequency and time domains, respectively. At the $T_{n}$ th OFDM symbol, the number of pilot subcarriers is defined as $<_{\mathbf{n}}=\left|\mathcal{F}_{\mathbf{n}}\right|$. The pilot inserted OFDM symbol is transmitted over the wireless channel after performing an inverse fast Fourier transform (IFFT) and adding a CP. It is assumed that the length of $\mathrm{CP},=\mathbf{f}$, is longer than the channel maximum delay, $=\mathrm{a} h$, making the channel matrix circulant $(=\mathrm{a} h \leq=\mathbf{f})$.

At the receiver, after perfect synchronization, the removal of $\mathrm{CP}$, and FFT operation, the received pilot symbol for the dth receive antenna can be represented as

$\mathbf{y}_{\mathrm{o}}\left[\mathrm{T}_{\mathrm{n}}\right]=\operatorname{LPK} 0\left(\mathbf{x}_{\mathrm{n}}\right) \mathbf{F}_{\mathrm{n}} \mathbf{h}_{\mathrm{n}, \mathrm{o}}+\mathbf{n}_{\mathrm{o}}$,

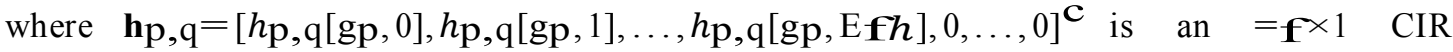
vector at the ath transmit antenna and dth receive antenna. ()$^{\mathrm{S}}$ and $(\cdot)=$ represent the transpose operation, and the transpose and conjugate op-

eration of a vector or matrix, respectively. $\mathbf{x}_{n}\left[={ }_{n}\left[\mathrm{P} 1, \mathrm{~T}_{\mathrm{n}}\right], \mathrm{n}_{\mathrm{n}}\left[\mathrm{P} 2, \mathrm{~T}_{\mathrm{n}}\right], \ldots, \mathrm{n}_{\mathrm{P}} \mathrm{PH}_{\mathbf{r}}, \mathrm{T}_{\mathrm{n}}\right] \mathrm{S}$ denotes a pilot vec-tor at the $\mathrm{T}_{\mathrm{n}}$ th OFDM symbol for $\mathrm{P} \mathbf{i} \in \mathcal{F}_{\mathrm{n}}$ and $\mathrm{R}=1,2, \ldots,<_{\mathrm{n}}$.

KDI and ME: PILOT-SYMBOL ASSISTED POWER DELAY PROFILE ESTMCATION FOR MMNO-OFDM SYSTEMS

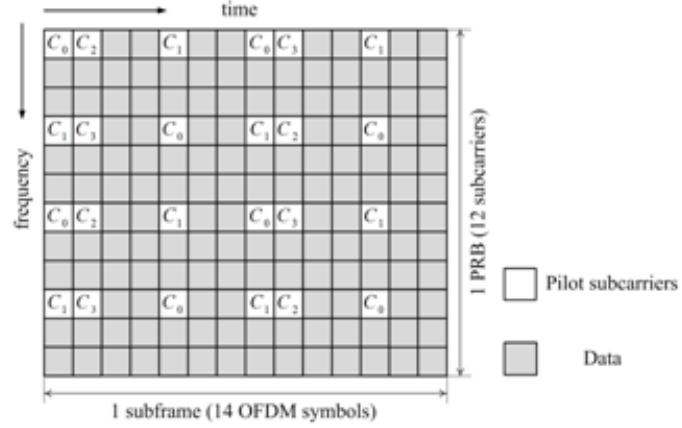

Fig. 1. Pilot symbol arrangement in a physical resource block (PRB) of the LTE OFDM system.

$\operatorname{LPKO}\left(\mathbf{x}_{n}\right)$ is the $<_{n} \times<_{n}$ diagonal matrix whose entries are the $<_{n}$ elements of the vector $x_{n}$. $F_{h}$ is a $<_{n} \times=_{f}$ matrix with the $\left(P_{i}, 5\right)$ th entry $\left[F_{n}\right]_{, j}=1 / N_{j a}\left\{-02 p P_{i} S<\right\}$ where $P_{i \in \mathcal{F}_{n}}$ and $S=0,1, \ldots,=\mathrm{n}_{\mathrm{f}}$ is a complex additive white Gaussian noise (AWGN) vector at the dth receiver antenna with each entry having a zero-mean and variance of $q^{2}$

III. Proposed Method For the PDP Estimation A. Derivation of the PDP in MIMO-OFDM systems

From (1), the CIR at the $(a, d)$ th antenna port can be estimated approximately using the regularized least squares (RLS) channel estimation with a fixed length of $=_{f}$ as

$$
\begin{aligned}
& \hat{\mathbf{h}}_{Q_{n}, \Omega}=\left(\mathrm{F}_{0,}=\mathrm{F}_{n}+\mathbf{s}_{K_{*}}{ }^{-1} \mathrm{~F}_{n}^{=} \operatorname{LPKO}\left(\mathbf{x}_{n}\right)=\mathbf{y}_{0}\left[T_{n}\right]\right. \\
& \triangleq \mathbf{W}_{Q K R_{, n}, \mathbf{y}_{0}\left[T_{n}\right]}
\end{aligned}
$$

where_ $Q(A)$ is the column vector containing all the diagonal elements of A. Then, the relation in (4) is simplified as

$$
\mathbf{p}_{\lambda}=a_{0} \mathbf{t}_{0}+a_{1} \mathbf{t}_{1}+\ldots+a_{K_{k}-1} \mathbf{t}_{K_{k}-1} \triangleq \mathbf{T p}_{\AA}
$$

where $\mathbf{T}=\left[\mathbf{t}_{0}, \mathbf{t}_{1}, \ldots, \mathbf{t}_{K_{k}-1}\right]$ is defined as a distortion matrix by $\mathbf{W}$. It is noted that the distortion matris a strictly diagonally dominant matrix, satisfying $[T]_{g g}>>_{b=\gamma}[T]_{g h} /$ for all $P, Q$, since the non-diagonal elements of $T$ are composed of the leakage powers of $\mathbf{u}_{g}$ for all $P$. From the Gershgorin circle theorem, a strictly diagonally dominant matrix is non-singular [13]. In addition, the distortion matrix is a well-conditioned matrix. Hence, the distortion of $\mathbf{W}$ can be eliminated as

$$
\mathbf{p}_{h}=\mathbf{T}^{-1} \mathbf{p}_{\lambda}^{n}={ }_{-}\left\{g_{n, 0}\left[T_{n}\right]\right\}-q^{2} w^{,}
$$

where $g_{n, 0}\left[T_{n}\right]=T^{-1}{ }_{0} \quad \mathbf{m}, n, 0 \hat{\mathbf{h}, n, 0}$ is defined as the received sample vector for estimating PDP at the $(a, d)$ th antenna port on the $T_{n}$ th OFDM symbol, and $\mathbf{w}=$ $\mathbf{T}^{-1} \mathrm{O}\left(\mathbf{W}_{Q K R, n} \mathbf{W}_{Q \bar{K} R, n}\right)$.

\section{B. PDP Estimation in Practical MMMO-OFDM Systems}

The received sample vector in (6) can be expressed as

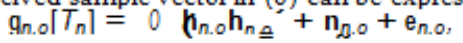

where $\mathbf{n}_{n, Q}=\mathbf{T}^{-1} \_\left(\mathbf{W}_{Q K R, n} \mathbf{n}_{0} \mathbf{n}=\mathbf{W}_{Q \overline{K R}, n}\right)$ and $\mathrm{e}_{n, 0}=$ $2 E N\left\{\mathbf{T}^{-1} \rho\left(\mathbf{W h}_{n, 0} \mathbf{n}=\mathbf{W}_{O K R}\right)\right\}$. Heqre, $E N\{\mathrm{a}\}$ denotes the real part of $\mathbf{a}$. We assume that $\boldsymbol{n}_{n, a}$ is an effective noise by AWGN. Then, the sample average of $g_{n, 0}\left[T_{n}\right]$ is given by

$$
\begin{aligned}
& \text { עr } \sum^{N} \sum^{P} \\
& \left\langle g_{n, 0}\left[T_{n}\right]\right)_{L} \triangleq \top \sum_{L=1}^{J r} \sum^{N} \sum_{\infty=1}^{P} g_{n, 0}\left[T_{n}\right]
\end{aligned}
$$

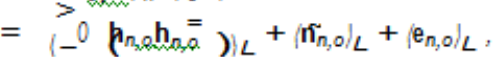


where $s=0.001$ is a small regularization parameter, and $\mathbf{I}_{K_{k}}$ is the $=_{f} \times=_{f}$ identity matrix. $F_{n} F_{0}$ in (2) is ill-conditioned due to the sparsity of pilot tones in the frequency domain and the presence of virtual subcamiers [8]. To derive the PDP from the estimated CIR in (2), the ensemble average of $h_{\text {Q.ang }} \boldsymbol{h}_{n} \mathrm{n}$ is given by

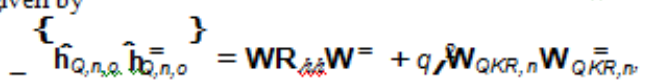

where $\mathbf{R}_{b k}=\left\{\mathbf{h}_{n, 0} \mathbf{h}_{n, 0\}}=\mathbf{a n d} \mathbf{W}=\left(\mathbf{F}_{n} \mathbf{F}_{n}+\mathbf{s} /_{K_{k}}\right)^{-1} \mathbf{F}_{n} \mathbf{F}_{n}\right.$ Note that the diagonal elements of the channel covariance matrix, $\mathbf{R}_{\text {s }}$ represent the PDP of multipath channel within the length of $\boldsymbol{=}_{\boldsymbol{f}}$, and all off-diagonal elements are zeros. Hence, the covariance matrix can be expressed as $\mathbf{R}_{h b}=L P K O\left(\mathbf{p}_{h}\right)$, where $\mathbf{p}_{h}=\left[a_{0}, a_{1}, \ldots, a_{K_{\text {nos }}}, 0, \ldots, 0\right]^{3}$ and $a_{j}=\left\{\left\{h_{n, 0}\left[T_{n}, j\right]\right\}\right.$. Unfortunately, $\mathbf{R}_{b,}$ is distorted by $\mathbf{W}$, which is an ill-conditioned matrix due to the presence of $F_{0}^{=} F_{n}$. Thus, instead of calculating $\mathbf{W}^{-1}$, we investigate the method for eliminating the spectral leakage of $\mathbf{W}$.

The covariance matrix of the estimated CIR is defined as $\mathbf{R}_{\sim \sim}=\mathbf{W R}_{\boldsymbol{b}} \mathbf{W}^{=}$which can be expressed as

的

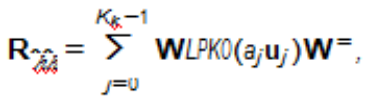

where $\mathbf{u}_{j}$ is a unit vector with the thentry being one and otherwise zeros. Let $\mathbf{p}_{n}$ and $\mathbf{t}_{j}$ be the $=_{f} \times 1$ vectors defined as $\mathbf{p}^{n}=\_\left(\mathbf{R}_{\sim}\right)$ and $\mathbf{t}_{j}=\_0 \mathbf{W} \operatorname{W} P K O\left(\mathbf{u}_{j}\right) \mathbf{W}^{=}$, respectively,

$i$ in

where $0 j_{, 0}\left[T_{n}\right]=\left[g_{n, 0}\left[T_{n}\right]\right]$ and $\tilde{\boldsymbol{\gamma}}=[\mathbf{w}]_{i,}$. To mitigate the detrimental effect of residual noise $\mathbf{z}_{L}$, the proposed scheme estimates the average of residual noise at the zero-taps of $\mathbf{p}$ At the sth entry of $\mathbf{p}_{q} / g$, the zero-tap can be detected as

$$
\psi_{t}^{j}=\begin{array}{cc}
1 & \text { if } \hat{a} i_{g r r}<n_{k b} \\
0 & \text { otherwise }
\end{array},
$$

where $n_{c k}=\frac{1}{K_{k}} \sum_{K_{k}-1} \hat{a}_{i j g r}$ is defined as a threshold value for the zero-tap detection. Then, the average of residual noise at the zero-taps can be estimated as

$$
T_{Q, T s f}=\frac{1}{>_{t}}{ }_{j=0}^{K} \hat{a}_{\xi / g r}^{i} h_{t}^{j}
$$

where $>_{t}=\sum_{K_{k}-1} h^{j}$ represents the total number of detected zero-taps. With the mitigation of residual noise, the th tap of the PDP estimate, $\hat{\mathbf{p}}_{\hat{h}}$, can be expressed as

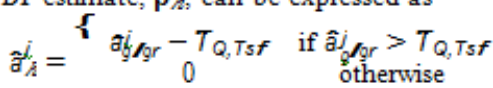

Then, the estimated PDP in (15) can be used to obtain the frequency-domain channel correlation in the LMMSE channel estimator.

IV. PERFORMANCE AND COMPLEXITY ANALYSIS

The LMMSE channel estimator with the imperfect PDP in (15) is given by

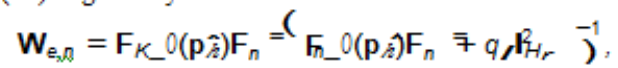

where $F_{K}$ is the $<_{c} \times=_{f}$ matrix obtained by taking the first ${ }_{f} \boldsymbol{f}$ columns of the DFT matrix. $\hat{\mathbf{p}} h=\mathbf{p}_{h}+\overline{\mathrm{e}}_{n \in n}$ is expressed as the estimated PDP, where the th element of $\operatorname{san}_{\text {n }}$ is defined where $>\triangleq L_{n} \mid C D$ represents the totalnumber of samples for PDP estimation. $L_{n} /$ is the number of pilot symbols at the $R_{n}$ th subcarier in a time slot. When $>$ is; suffipiently large, the PDP can be perfectly estimated, since $-0 \mathbf{h}_{n, 0} \mathbf{h}_{0,0} \rightarrow \mathbf{p}_{h}$ $\left\langle\tilde{n}_{n, 0}\right\rangle_{L} \rightarrow q_{\ell}^{2} \tilde{W}$, and $\left\langle\mathbf{e}_{n, 0}\right\rangle_{L} \rightarrow 0$. However, it is difficult for a receiver of practical MIMO-OFDM systems to obtain such a large number of samples. With an insufficient number of sampley, the PDP can be approximated as $\mathbf{p}_{h} \approx$

- $\mathbf{h}_{n, 0} \mathbf{h}_{n, 0}=$

To improve the accuracy of PDP estimation with insufficient samples, we mitigate the effective noise as follows

$$
\left(q_{n . o}\left[T_{n}\right]\right),-q, \mathbf{w}=0 \mathbf{h}_{(0} \mathbf{h}_{n}=+\mathbf{z}_{L} \text {, }
$$

where $\mathbf{z}_{L} \triangleq\left(\mathrm{e}_{n, a}\right)+\left(\mathbf{m}_{n, 0}\right)_{L}-q 3 \mathbf{w}$ is defined as a residual noise vector, in which each entry has a zero-mean. Then, the error of PDP estimation with $>$, samples can be calculated as $\mathrm{e}_{L}=\left(0 \mathrm{~h}\left(\mathrm{foh}_{0} \mathrm{~h}_{0}-\mathrm{p}_{\ell}\right)+\mathrm{z}_{L}\right.$. (10) Since $\left[\mathbf{p}_{h}\right]_{g} \geq 0$ for all $P$, the PDP can initially be estimated as

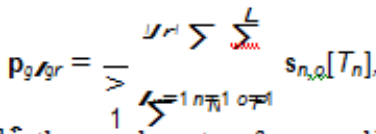

where $\mathbf{s}_{n, 0}\left[T_{n}\right]$ is the sample vector of proposed PDP estimator with the Sth entry

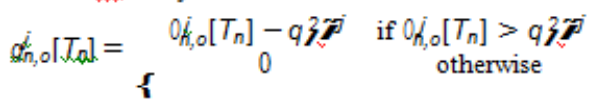

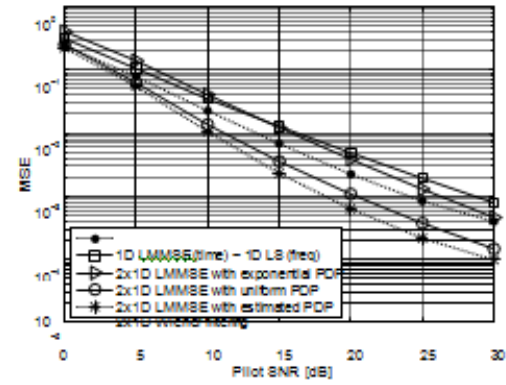

Fig. 2. Performance of LMMSE technique using the estimated PDP over ETU channel.

where ${ }^{\wedge} \mathbf{h}_{K R, \Omega \Omega} \triangleq \operatorname{LPKO}\left(\mathbf{x}_{n}\right)^{=} \mathbf{y}_{n}\left[T_{n}\right]$. Using the error covari-ance matrix, the frequency-domain MSE of the proposed scheme is given by

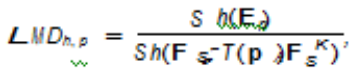

where $\mathrm{He}\left(\mathbf{E}_{n}\right)$ denotes the trace operation of $\mathbf{E}_{n}$. With a sufficiently large number of samples, encon $\rightarrow 0$. Thus, the MSE of the proposed scheme achieves that of Wiener filtering because $\mathbf{W}_{\mathrm{e}, \Omega} \rightarrow \mathbf{W}_{\mathrm{s} r, \mathrm{n}, \mathrm{.}}$

The additional complexity by the proposed PDP estimation technique is $B==_{f}+<_{n}={ }_{f}+L_{n} C D==_{f}$, which mainly comes from computing (2) and (6). When the pilot spacing is fixed in the frequency domain, all entries of $\boldsymbol{r}_{n}$ and $\mathbf{I}$ are constant. Thus, $\left(F_{n} F^{=}+s \mathbf{I}_{k}\right)^{-1} F_{n}^{=}$and $\mathbf{T}^{-1}$ can be computed only once, and their values chn be stored. The additional complexity is then reduced to $B=f+I_{n} \mid C D={ }_{f}$. 


$$
P_{n c n}^{i}=\mathbf{\{} \begin{array}{cc}
{\left[\mathrm{e}_{L}\right]_{j}-T_{Q, T s f}} & \text { if }\left[\mathrm{e}_{L}\right]_{j}>T_{Q, T s f} \\
0 & \text { otherwise }
\end{array}
$$

From the matrix inversion lemma, $\left(\mathbf{F}_{p}-T\left(\hat{p}_{i}\right) \mathbf{F}_{p}{ }^{\kappa}+n_{0} \hat{\mathbf{1}}_{L_{r}}\right)-$ in (22) is converted as

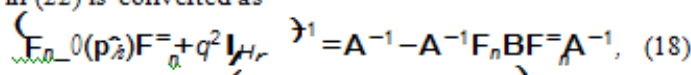

$$
\begin{aligned}
& \text { where } \left.A_{(} \triangleq F_{n} O\left(p_{\beta}\right) F_{0}=q^{2} \mathbf{I}_{H_{r}}\right) \text { and } \mathrm{B}
\end{aligned}
$$

Then, the coeffi-cient matrix for LMMSE channel estimation with $\mathbf{p}_{\delta}$ can be rewritten as

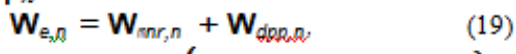

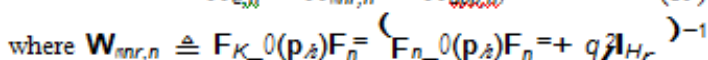
is the coefficient matrix for Wiener filtering, and $\mathbf{W}_{\phi i g h}$ is given by

$$
\begin{aligned}
& \mathbf{W}_{\text {dig口, }}=-\mathrm{F}_{K_{-}} \mathrm{O}\left(\mathrm{p}_{h}\right) \mathrm{F}^{=} \mathrm{A}^{-1} \mathrm{~F}_{n} \mathrm{BF}=\mathrm{A}^{-1}
\end{aligned}
$$

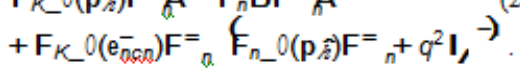

The error covariance matrix of LMMSE channel estimation with the imperfect PDP can be obtained as

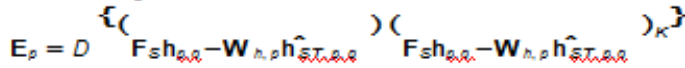

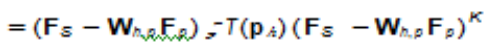

$$
\begin{aligned}
& +n_{p}^{2} \mathbf{W}_{m, \rho} \mathbf{F}_{p} \mathbf{F}_{p}{ }^{K} \mathbf{W}_{n, p}^{K}
\end{aligned}
$$

\section{Simulation Results}

We consider a MIMO-OFDM system with the physical layer parameters for the downlink of 3GPP LIE [14]. The system bandwidth is 5 MHz with 301 subcarriers for transmitting data information and pilots including a DC subcarrier at 2--- $k$ carrier frequency. The width of each subcarrier is $15 \mathrm{kHz}$ with an FFT size of 512 . The MIMOOFDM system utilizes four transmit and two receive antennas $(C=4, D=2)$. We assume that the pilots of the four transmit antenna ports are distributed as the time and frequency grid of the LIE system in Fig. 1. The length of CP is 40 $(=f=40)$. For all simulations, the channel estimator is based on a cascaded $2 \times 1 D$ LMMSE technique during 14 OFDM symbols $\left(l_{1}|=| L_{2}|=2,|{ }_{3}|=|{ }_{4} \mid=1\right)$, as shown in Fig. 1 , where the filtering in frequency domain is followed by the filtering in time domain over slowly fading channels with the Doppler frequency of $5 \mathrm{~Hz}$.

Figure 2 shows the MSE performance of the $2 \times I D$ LMMSE technique using the estimated PDP. All underlying links are modeled as extended typical urban (ETU) channels [14]. The performance of the $2 \times 1 D$ Wiener filter with exact $P D P$ is included as a lower bound. For performance comparisons, we plot the performance of frequency domain regularized LS channel estimation in which the PDP information is not required. The performance of the $2 \times 1 D$ LMMSE technique using the approximated PDP, which is uniform or exponential model with the channel delay parameter estimation in [11], is also plotted. Note that the LMMSE technique using the estimated PDP outperforms the conventional methods, since the correlation mismatch is reduced by the proposed PDP

KNG and DN: PLOT-SYMBOL ASSISTED POWER DELAY PROFLE ESTIMATION FOR MINO-OFDM SYSTEMS

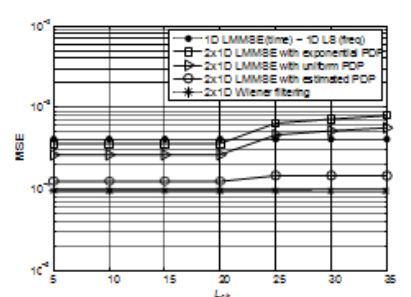

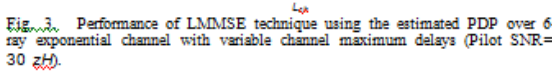

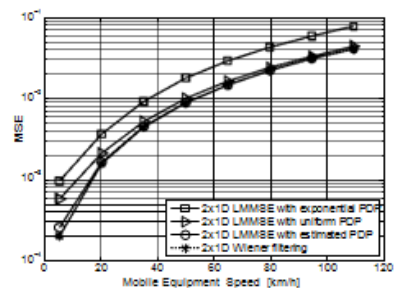

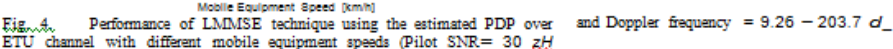

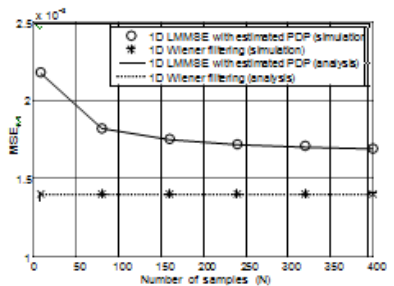

Eig 5 . Simulation and analysis results of LMMSE channel estimation Fver ETU channel with various number of samples for the PDP estimation
(Pilot SNR $=20 \mathrm{z}$ ).

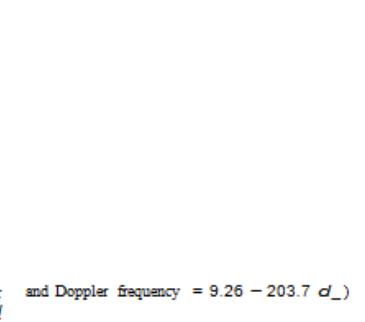

We also observe from Fig. 3 that the proposed method has a performance loss within only a 2.4-dB gap, compared with $2 \times 1 \mathrm{D}$ Wiener filtering.

In Fig. 3, we investigate the MSE performance of the pro-posed scheme over the exponentially power 
decaying six-path Rayleigh fading channel model, where the channel maximum delay, $={ }_{a h}$, is variable. The PDP of the channel model is de-fined as $\left.{ }_{-}\left\{/ \boldsymbol{Z}^{j}{ }_{, o}[T]\right\}^{2}\right\}={ }^{1} N^{j / v_{s P}}$ for $S=0, \Delta r, n ., 5 \Delta r$ and $\Delta r={ }_{5}^{K}{ }^{h}$. Here, $\left.r_{p} \boldsymbol{k} q={ }_{j m f(2 K}\right)$ and $G_{h}$ is the normalization

factor $\left(G_{h}=\frac{K_{h h}}{j^{J_{s p}}}\right)$. The performance of the proposed scheme is better than that of the conventional methods, and

approaches that of Wiener filtering in various channel envi-ronments.

Figure 4 shows the MSE performance of the $2 \times 1 \mathrm{D}$ LMMSE technique using the estimated PDP for different mobile equip-ment speeds at 30-L_ SNR. All underlying links are modeled as ETU channels. In Fig. 4, it can be seen that the MSE of LMMSE technique using the estimated PDP achieves that of Wiener filtering even at high Doppler frequencies.

Figure 5 shows simulation and analysis results of the frequency-domain LMMSE channel estimation with various samples for obtaining the PDP at $20-L_{-}$SNR $\left(>=L_{n} / C D\right)$. We assume that $2 \times 2$ MIMO-OFDM system over ETU chan-nels with $70-,-k$ Doppler frequency. The simulation results correspond to the channel estimation performance at the first OFDM symbol of antenna port 1 shown in Fig. 1. We obtain the analytic results in (22) by using the coefficient matrix for LMMSE channel estimation with the perfect or imperfect PDP at the antenna port. In Fig. 5, it is observed that the MSE of the proposed scheme improves the MSE performance with an increase in the number of samples for PDP estimation.

\section{Conclusions}

We proposed a PDP estimation technique for the LMMSE channel estimator in MIMO-OFDM systems. The CIR esti-mates at each path of the MIMO channels were used to obtain the PDP. For accurate PDP estimation, we considered the spectral leakage effect from virtual subcarriers, and the resid-ual noise caused by the insufficient number of estimated CIR samples. The proposed technique effectively mitigates both the spectrum leakage and residual noise. Simulation results show that the performance of LMMSE channel estimation using the proposed PDP estimate approaches that of Wiener filtering.

\section{References}

[1] TS 36.211, "Physical channels and modulation," in 3GPP TSG RAN1, Rev. 10.1.0, Jan. 2011.

[2] T. W. Yune, C. H. Choi, G. H. Im, J. B. Lim, E. S. Kim, Y. C. Cheong, and K. H. Kim, "SC-FDMA with iterative multiuser detection: improvements on power/spectral efficiency," IEEE Commun. Mag., vol. 48, no. 3, pp. 164-171, Mar. 2010.

[3] U. K. Kwon, D. Kim, and G. H. Im, "Amplitude clipping and iterative reconstruction of MIMO-OFDM signals with optimum equalization,” IEEE Trans. Wireless Commun., vol. 8, pp. 268-277, Jan. 2009.

[4] L. Zheng and D. N. Tse, "Diversity and multiplexing: a fundamental tradeoff in multiple antenna channels," IEEE Trans. Inf. Theory, vol. 49, pp. 1073-1096, May 2003.

[5] H. C. Won and G. H. Im, "Iterative cyclic prefix reconstruction and channel estimation for a STBC OFDM system," IEEE Commun. Lett., vol. 9, pp. 307-309, Apr. 2005.

[6] T. Yoo and A. Goldsmith, "Capacity and power allocation for fading MIMO channels with channel estimation error," IEEE Trans. Inf. Theory, vol. 52, pp. 2203-2214, May 2006.

[7] M. G. Song, D. Kim, and G. H. Im, "Recursive channel estimation method for OFDM-based cooperative systems," IEEE Commun. Lett., vol. 14, no. 11, pp. 1029-1031, Nov. 2010.

[8] S. Omar, A. Ancora, and D. T. M. Slock, "Performance analysis of general pilot-aided linear channel estimation in LTE OFDMA systems with application to simplified MMSE schemes," in Proc. IEEE PIMRC 2008.

[9] T. Cui and C. Tellambura, "Power delay profile and noise variance estimation for OFDM," IEEE Commun. Lett., vol. 10, pp. 25-27, Jan. 2006.

[10] X. Gong, C. Zhao, W. Xu, and M. Jiang, "Power delay profile estimation for MIMO-OFDM systems over time-varying multipath channels," in Proc. IEEE ICCT 2010.

[11] K. C. Hung and D. W. Lin, "Pilot-based LMMSE channel estimation for OFDM systems with power-delay profile approximation," IEEE Trans. Veh. Technol., vol. 59, pp. 150-159, Jan. 2010.

[12] Y. Li, J. H. Winters, and N. R. Sollenberger, "MIMO-OFDM for wireless communications: signal detection with enhanced channel estimation," IEEE Trans. Commun., vol. 50, pp. 1471-1477, Sep. 2002.

[13] R. Horn and C. Johnson, Matrix Analysis. Cambridge University Press, 2009.

[14] TS 36.101, "User equipment (UE) radio transmission and reception," in 3GPP TSG RAN1, Rev. 10.1.0, Jan. 2011. 